\title{
Applications of the Gurson's model in sheet metal forming
}

\author{
Dorel Banabic ${ }^{1, *}$ and Abdolvahed $\mathrm{Kami}^{2}$ \\ ${ }^{1}$ Manufacturing Engineering Department, Technical University of Cluj Napoca, Memorandumului 28, Cluj Napoca, Romania \\ ${ }^{2}$ Mechanical Engineering Department, Semnan University, Semnan, Iran
}

\begin{abstract}
Recent advances in the modelling of metals encompass modelling of metals structural inhomogeneity, damage, porosity, twinning/untwining and non-local and second order effects. This presentation is focused on modelling the void growth in ductile fractures. The growth and coalescence of microscopic voids are the main mechanisms in ductile fracture of bulk metallic parts. In sheet metals, ductile fracture is preceded by necking during which existing voids do not have significant growth. However, necking is highly sensitive to plastic flow direction which in turn is sensitive to the presence of voids. Also, under biaxial strain loading, the final fracture in the necking region is still controlled by void growth; hence an accurate fracture prediction is crucial for crash simulations. Finally, in super-plastic sheet forming, void growth and coalescence may precede or accompany necking. Therefore, there is as increasing interest in modelling of voids in the sheet metals. As an application, we show how the predictions of some forming limit curves (FLCs) can be affected by accurate simulation of voids growth.
\end{abstract}

Keywords: Ductile fracture, Sheet metal, Forming limit curve

\section{Introduction}

Fracture in ductile metallic materials is due to evolution of tensile load-induced voids. During plastic deformation, new voids will nucleate around inclusions and second phase particles. These voids grow along with the initial voids in the material structure and finally coalesce. As a result, micro-cracks will be formed and material fracture will occur. This procedure is schematically illustrated in Fig. 1.

The first works on the voids growth were conducted by McClintock [1] and Rice and Tracey [2]. They studied the voids growth in a rigid-plastic infinite medium. Later, Gurson [3] proposed a new model for porous ductile materials which predicted both the growth of such voids and their effect on the yield criterion. The Gurson's model was modified by considering models for void nucleation and coalescence [4-6]. This modified model is known as Gurson-Tvergaard-Needleman (GTN) model which has been widely adopted in the engineering community during last 30 years [7].

The sheet metal exhibit a significant anisotropy due to the rolling process used in their production procedure. So, to apply Gurson type models on sheet metal, it is important to consider plastic anisotropy. Benzerga and Besson [8] extended Gurson's model for a material matrix which follows Hill's orthotropic criterion [9]. Later, Monchiet et al. [10,11] and Keralavarma and Benzerga $[12,13]$ extended the anisotropic Gurson model to spheroidal voids. Recently, Morin et al. [14] developed a Gurson type model for general ellipsoidal voids in an anisotropic Hill matrix. Stewart and Cazacu [15] extended
Gurson's model for anisotropic porous aggregates which display tension-compression asymmetry (e.g. metals with hexagonal crystal structure) and contain spherical voids. The material matrix behaviour can be defined by the anisotropic Cazacu et al. [16] yield criterion [7].

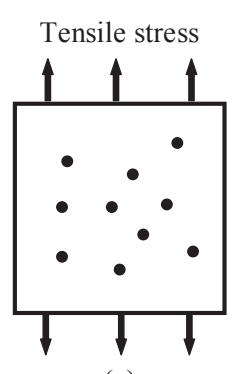

(a)

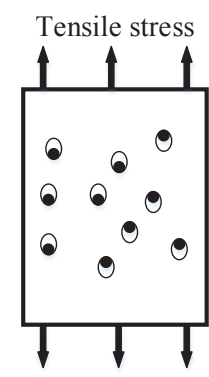

(b)

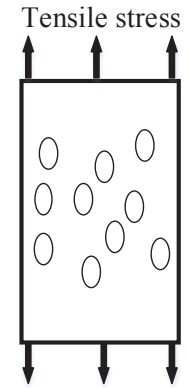

(c)

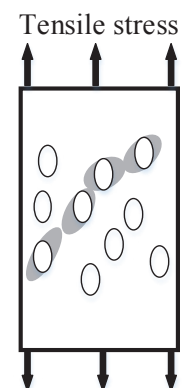

(d)

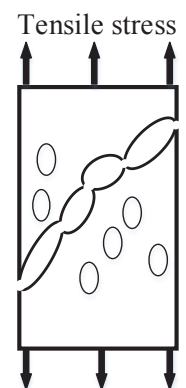

(e)
Fig. 1. Evolution of the voids and consequent fracture of sheet metal under tensile loading, (a) initial material with second phase particles and inclusions, (b) nucleation of new voids, (c) voids growth, (d) onset of necking between voids and (f) voids coalescence and sheet metal fracture.

\footnotetext{
* Corresponding author: banabic@tcm.utcluj.ro
} 
In contrary to bulk components where ductile failure is clearly due to the void evolution, sheet metals fail mainly through necking. The voids can influence the normal to the yield criterion well before necking, which affects the prediction of forming limits. Also, voids provide the final rupture mechanism in the necking region, especially under biaxial strain conditions [7].

Assuming that voids are spherical and remain so during deformation, the main problem is to include GTN in anisotropy of material matrix. Most of studies have been focused on Hill'48 quadratic anisotropy (e.g. [8, 17]). Liao et al. [18] derived an approximate potential close to the original Gurson formulation for anisotropic sheets containing thickness holes. Wang et al. [19] modified Liao et al. model by using average anisotropy parameter in the constitutive equations. Chen and Dong [20] extended the GTN model to characterize the material matrix through Hill quadratic and Barlat-Lian 3component [21] expressions of the equivalent stress.

In this paper, to explore the role of voids in sheet metal formation, the implementation of a simple anisotropic GTN model was implemented in ABAQUS. Then, the results of the numerical simulation of a deep drawing process were presented by GTN model. Finally, the results of numerical simulation of Nakazima tests with GTN model and its application in calculation of FLCs of single and sandwich sheets were provided.

\section{GTN model}

As mentioned in the previous section, different extensions of the original GTN model [4-6] have been presented considering the anisotropic behaviour of the material matrix. A common one is the extension which uses Hill'48 yield criterion to describe material matrix yielding. In this section, a brief description of this GTN extension will be presented. In the next section, its implementation in ABAQUS/Explicit code will be described.

The potential function of this model is as follows [17]:

$$
\Phi=\left(\frac{\bar{\sigma}}{Y}\right)^{2}+q_{1} f^{*}\left[2 \cosh \left(-q_{2} \frac{3 p}{2 Y}\right)-\frac{q_{3}}{q_{1}} f^{*}\right]-1
$$

where $\bar{\sigma}$ is the Hill' 48 equivalent stress, $Y$ is the yield stress of the matrix material, $p$ is hydrostatic pressure, $q_{l}$, $q_{2}$ and $q_{3}$ are adjustment parameters and $f^{*}$ is a quantity, which depends on the current value of void volume fraction. This quantity is defined as Eq. (2).

$$
f^{*}=\left\{\begin{array}{llr}
f, & \text { if } \quad f \leq f_{c}, \\
f_{c}+\frac{f_{f}^{*}-f_{c}}{f_{f}-f_{c}}\left(f-f_{c}\right), & \text { if } f_{c}<f<f_{f}, \\
f_{f}^{*}, & \text { if } \quad f \geq f_{f}
\end{array}\right.
$$

All admissible stress states of the sheet metal are defined by the $\Phi \leq 0$ inequality. Accurately, when $\Phi<0$ defines the elastic states and $\Phi=0$ indicates to elastoplastic states. Accordingly, the flow rule associated to the GTN potential can be expressed in the following form:

$$
\dot{\varepsilon}^{(p)}=\dot{\lambda} \frac{\partial \Phi}{\partial \sigma}, \quad \text { where } \begin{cases}\dot{\lambda}=0, & \text { if } \Phi<0, \\ \dot{\lambda} \geq 0, & \text { if } \Phi=0 .\end{cases}
$$

where, $\dot{\varepsilon}^{(p)}$ is the plastic part of the strain rate tensor.

The change of the void volume fraction $f$ is caused by the growth of the initial void volume fraction $f_{0}\left(\dot{f}^{(g)}\right.$ ) and the nucleation of new voids at the limits of the second phase particles and inclusions $\left(\dot{f}^{(n)}\right)$ :

$$
\dot{f}=\dot{f}^{(g)}+\dot{f}^{(n)}
$$

where

$$
\dot{f}^{(g)}=(1-f)\left[\dot{\varepsilon}_{11}^{(p)}+\dot{\varepsilon}_{22}^{(p)}+\dot{\varepsilon}_{33}^{(p)}\right]
$$

and

$$
\dot{f}^{(n)}=\frac{f_{N}}{S_{N} \sqrt{2 \pi}} \exp \left[-\frac{1}{2}\left(\frac{\bar{\varepsilon}^{(p)}-\bar{\varepsilon}_{N}}{S_{N}}\right)^{2}\right] .
$$

The quantities denoted as $S_{N}, \bar{\varepsilon}_{N}$ and $f_{N}$ in Eq. (6) are also material constants that must be determined by identification.

The plastic strain be subdivided into volumetric and deviatoric parts. So, it is possible to derive the following formuations for the flow rule:

$$
\dot{\varepsilon}^{(p, d e v)}=\dot{\lambda} \frac{\partial \Phi}{\partial \bar{\sigma}}, \quad \dot{\varepsilon}^{(p, v o l)}=-\dot{\lambda} \frac{\partial \Phi}{\partial p},
$$

So, one can easily conclude that:

$$
\dot{\varepsilon}^{(p, d e v)} \frac{\partial \Phi}{\partial p}+\dot{\varepsilon}^{(p, v o l)} \frac{\partial \Phi}{\partial \bar{\sigma}}=0 .
$$

Eq. (8) will be used as a consistency condition in calculations of the state variables appearing in implementation of the GTN model.

\section{Implementation of GTN model}

The GTN damage model has been comibined with Abaqus/Explicit to perform simualtions of deformation. To this end, the damage model has been implemented in a VUMAT routine. Abaqus/Explicit divides nonlinear processes simulations into small $[t, t+\Delta t]$ time increments. The VUMAT routine recieves the components of the stress tensor ${ }^{t} \sigma_{i j}$, the equivalent plastic strain ${ }^{t} \bar{\varepsilon}^{(p)}$ and the void volume fraction ${ }^{t} f$ at the reference state (time $t$ ) and evaluates these parameters at the current state (time $t+\Delta t$ ) i.e. VUMAT evaluates ${ }^{t+\Delta t} \sigma_{i j},{ }^{t+\Delta t} \bar{\varepsilon}^{(p)}$ and ${ }^{t+\Delta t} f$. 
The ${ }^{t+\Delta t} \sigma_{i j}$ componenets can be expressed as [17]:

$$
\begin{aligned}
& { }^{t+\Delta t} \sigma_{i j}={ }^{t+\Delta t} \sigma_{i j}^{(e)}-\frac{E}{1+v} \\
& {\left[{ }_{t}^{t+\Delta t} \varepsilon_{i j}^{(p)}+\frac{v^{t+\Delta t}}{1-2 v}{ }_{t} \varepsilon_{\ell \ell}^{(p)} \delta_{i j}\right],}
\end{aligned}
$$

where

$$
{ }^{t+\Delta t} \sigma_{i j}^{(e)}={ }^{t} \sigma_{i j}+\frac{E}{1+v}\left[{ }^{t+\Delta t} \varepsilon_{i j}+\frac{v}{1-2 v}_{t}^{t+\Delta t}{ }^{t} \varepsilon_{\ell \ell} \delta_{i j}\right]
$$

and

$$
{ }_{t}^{t+\Delta t} \varepsilon_{i j}^{(p)}=\int_{t}^{t+\Delta t^{\tau}} \dot{\varepsilon}_{i j}^{(p)} \mathrm{d} \tau .
$$

The ${ }^{t+\Delta t} \sigma_{i j}^{(e)}$ are componenets of a stress tensor that would occur in the current configuration if the incremental logarithmic strain were purely elastic. At first ${ }^{t+\Delta t} \sigma_{i j}^{(e)}$ will be evaluated using Eq. (10) and then the corresponding pressure and equivalent stress will be calculated, respectively:

$$
\begin{gathered}
{ }^{t+\Delta t} p^{(e)}=-\frac{1}{3}{ }^{t+\Delta t} \sigma_{\ell \ell}^{(e)}, \\
{ }^{t+\Delta t} \bar{\sigma}^{(e)}=\sqrt{{ }^{t+\Delta t} \sigma_{i j}^{(e)} P_{i j k \ell}{ }^{t+\Delta t} \sigma_{k \ell}^{(e)}} .
\end{gathered}
$$

By placing the ${ }^{t+\Delta t} p^{(e)}$ and ${ }^{t+\Delta t} \bar{\sigma}^{(e)}$, it is possible to evaluate ${ }^{t+\Delta t} \Phi^{(e)}$ and to decide whether the increment of the logarithmic strain is elastic or elastoplastic. The value of ${ }^{t+\Delta t} \Phi^{(e)}$ is equal to [17]:

$$
\begin{aligned}
& { }^{t+\Delta t} \Phi^{(e)}=\left[\frac{{ }^{t+\Delta t} \bar{\sigma}^{(e)}}{{ }^{t} Y}\right]^{2}+q_{1}{ }^{t} f^{*} \\
& {\left[2 \cosh \left(-q_{2} \frac{3^{t+\Delta t} p^{(e)}}{2^{t} Y}\right)-q_{1}^{t} f^{*}\right]-1,}
\end{aligned}
$$

where

$$
{ }^{t} Y=Y\left[{ }^{t} \bar{\varepsilon}^{(p)}\right]
$$

and

$$
{ }^{t} f^{*}=\left\{\begin{array}{cc}
{ }^{t} f, & \text { if }{ }^{t} f \leq f_{C}, \\
f_{C}+\frac{f_{F}^{*}-f_{C}}{f_{F}-f_{C}}\left({ }^{t} f-f_{C}\right), & \text { if } f_{C}<{ }^{t} f<f_{F} \\
f_{F}^{*}, & \text { if }{ }^{t} f \geq f_{F}
\end{array}\right.
$$

are parameters associated to the reference configuration. If ${ }^{t+\Delta t} \Phi^{(e)} \leq 0$, the incremental logarithmic strain ${ }_{t}^{t+\Delta t} \varepsilon$ is purely elastic and the parameters of the current configuration can be set as follows: ${ }^{t+\Delta t} \sigma_{i j}={ }^{t+\Delta t} \sigma_{i j}^{(e)}$, ${ }^{t+\Delta t} \bar{\varepsilon}^{(p)}={ }^{t} \bar{\varepsilon}^{(p)}$, and ${ }^{t+\Delta t} f={ }^{t} f$. On the other hand, if ${ }^{t+\Delta t} \Phi^{(e)}>0$, the material evolves through elastoplastic states during the time interval $[t, t+\Delta t]$. In such a case, the plastic terms on the right-hand side of Eq. (9) are different from zero. These terms will be evaluated using a backward Euler approximation of Eq. (11):

$$
{ }_{t}^{t+\Delta t} \varepsilon_{i j}^{(p)} \approx^{t+\Delta t} \dot{\varepsilon}_{i j}^{(p)} \Delta t .
$$

Eq. (10) can be rewritten in the normalized form in terms of the plastic strain increments [17]:

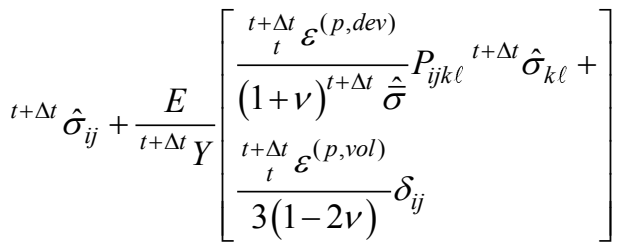

$$
\begin{aligned}
& \approx^{t+\Delta t} \hat{\sigma}_{i j}^{(e)},
\end{aligned}
$$

where

$$
\begin{aligned}
& { }^{t+\Delta t} Y=Y\left[{ }^{t+\Delta t} \bar{\varepsilon}^{(p)}\right], \\
& { }^{t+\Delta t} \hat{\sigma}_{i j}^{(e)}={\frac{1}{{ }^{t+\Delta t} Y}}^{t+\Delta t} \sigma_{i j}^{(e)}, \\
& { }^{t+\Delta t} \hat{\sigma}_{i j}={\frac{1}{{ }^{t+\Delta t} Y}}^{t+\Delta t} \sigma_{i j},
\end{aligned}
$$

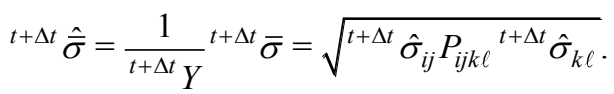$$
\text { And }{ }^{t+\Delta t} \varepsilon^{(p, d e v)} \text { and }{ }^{t+\Delta t} \varepsilon^{(p, v o l)} \text { are the deviatoric and }
$$

volumetric parts of plastic strain incerement (see Eq. (7)), respectively.

In the case of an elastoplastic evolution of the material, Eq. (18) is accompanied by the following constraints:

- First consistency condition, ${ }^{t+\Delta t} \Phi=0$ [17]:

$$
\begin{aligned}
& { }^{t+\Delta t} \hat{\bar{\sigma}}^{2}+q_{1} \\
& { }^{t+\Delta t} f^{*}\left[2 \cosh \left(-\frac{3}{2} q_{2}{ }^{t+\Delta t} \hat{p}\right)-q_{1}{ }^{t+\Delta t} f^{*}\right]=1,
\end{aligned}
$$

where

$$
{ }^{t+\Delta t} f^{*}=\left\{\begin{array}{cc}
{ }^{t+\Delta t} f, & \text { if }{ }^{t+\Delta t} f \leq f_{C}, \\
\left.f_{C}+\frac{f_{F}^{*}-f_{C}}{f_{F}-f_{C}}{ }^{t+\Delta t} f-f_{C}\right), & \text { if } f_{C}<^{t+\Delta t} f<f_{F}, \\
f_{F}^{*}, & \text { if }{ }^{t+\Delta t} f \geq f_{F},
\end{array}\right.
$$

and

$$
{ }^{t+\Delta t} \hat{p}=-\frac{1}{3}^{t+\Delta t} \hat{\sigma}_{\ell \ell}
$$

- Incremental form of the second consistency condition [17]:

$$
\begin{aligned}
& { }^{t+\Delta t} \hat{\bar{\sigma}}_{t}^{t+\Delta t} \varepsilon^{(p, v o l)}- \\
& \frac{3}{2} q_{1} q_{2}{ }^{t+\Delta t} f^{*} \sinh \left(-\frac{3}{2} q_{2}{ }^{t+\Delta t} \hat{p}\right)_{t}^{t+\Delta t} \varepsilon^{(p, d e v)} \approx 0
\end{aligned}
$$

The Eqs (18), (23) and (26) are a nonlinear set of equations having ${ }^{t+\Delta t} \hat{\sigma}_{i j},{ }^{t+\Delta t} \bar{\varepsilon}^{(p)}$ and ${ }^{t+\Delta t} f$ as unknowns. The VUMAT routine solves this set in a numerical manner, using a forward finite difference Newton scheme combined with a line search strategy. The reference state parameters ${ }^{t} \hat{\sigma}_{i j}={ }^{t} \sigma_{i j} /{ }^{t} Y,{ }^{t} \bar{\varepsilon}^{(p)}$ and ${ }^{t} f$ define the start point of the solution procedure. 


\section{Results and discussions}

The GTN model was used in ABAQUS/Explicit code to predict fracture of AA6016-T4 sheet in a square cup drawing simulation. Furthermore, the model was utilized for construction of FLCs of single AA6016-T4 sheet and $\mathrm{metal} /$ polymer/metal sandwiches.

\subsection{Prediction of fracture in deep drawing}

The GTN model was used for prediction of fracture in square cup drawing of 1-mm thick AA6016-T4 sheet. The mechanical properties of the sheet along with its Lankford's coefficients were obtained by conducting tensile experiments in $0^{\circ}, 45^{\circ}$ and $90^{\circ}$ directions with respect to the rolling direction. The Lankford's coefficients obtained from these experiments are $r_{0}=0.5529, r_{45}=0.4091$ and $r_{90}=0.5497$ [22]. The hardening behaviour of AA6016-T4 sheet can be described by Swift's law:

$$
Y=525.8\left(0.0113+\bar{\varepsilon}^{p}\right)^{0.27}
$$

Fig. 2 illustrates the fracture of the metallic sheet during the square cup drawing tests performed with a blank holding force of $10 \mathrm{kN}$. This figure also presents a comparison between the predictions of the GTN model with the experiments.

As Fig. 2 shows, the fracture path is almost the same in both images. In FE simulation with ABAQUS/Explicit and GTN model, the cup depth at the onset of fracture was predicted as $17.90 \mathrm{~mm}$ which is in a good agreement with the depth of $18.70 \mathrm{~mm}$ in the experimental specimen.

\subsection{FLC construction for single sheets}

The GTN model was also used for construction of FLC of AA6016-T4 sheet. For this purpose, FE simulations of Nakazima tests were conducted using ABAQUS/Explicit and GTN model. The simulations were performed on different notched specimens with different widths. These simulations were allowed to continue until the fracture emergence. Then, the major and minor limit strains were measured at five paths normal to the fracture line. The Bragard's method $[23,24]$ was utilized to find the limit strains at the onset of necking. The accuracy of the simulation results was validated by their comparison with the experimental tests of Nakazima tests.

Fig. 3 compares GTN-predicted FLC with the experimental FLC. As the figure depicts, GTN model provides good predictions for the limit strains in almost all areas of FLC. This indicates that the anisotropic GTNHill' 48 model has the capability of predicting limit strains for almost all forming paths.

For better understanding of GTN model capability on prediction of limit strains, FLC of AA6016-T4 sheet was calculated using M-K and MMFC models; as shown in Fig. 3. Although M-K and MMFC models provided good predictions for the left branch and middle section of FLC, they failed to predict the accurate limit strains on the right branch of AA6016-T4 sheet. On the other hand, GTN model presented good predictions for both the left and right branches of the FLC. This observation indicate that although GTN model has more computational cost, it is more powerful than M-K and MMFC models in prediction of FLC of AA6016-T4 sheet [17].

(a)

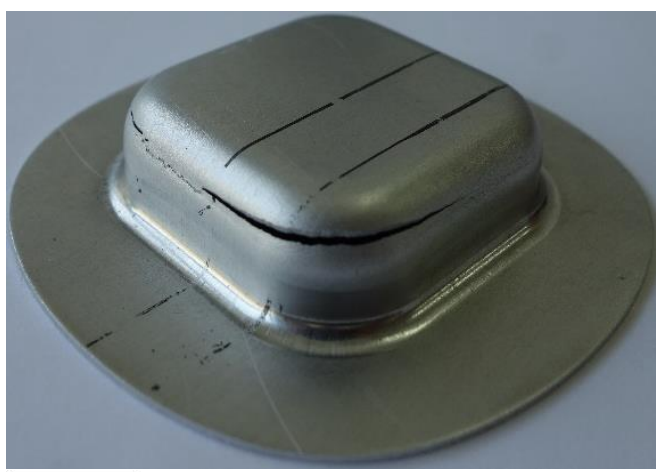

(b)

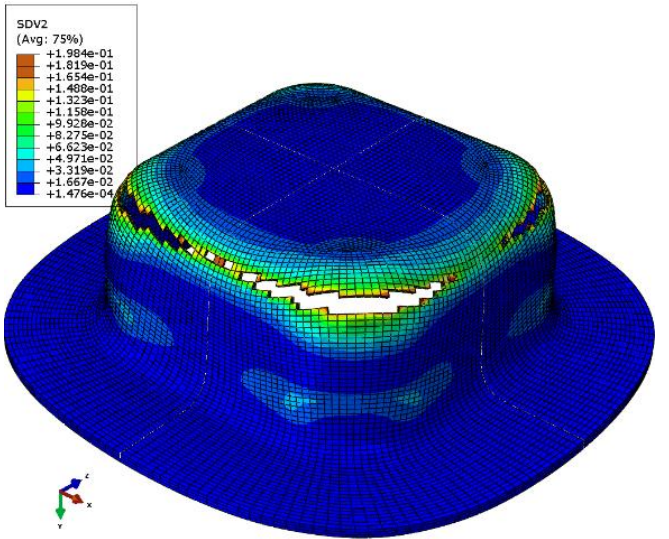

Fig. 2. Comparison of fractured specimens of the square cup drawing (a) experiment (b) FE simulation [22].

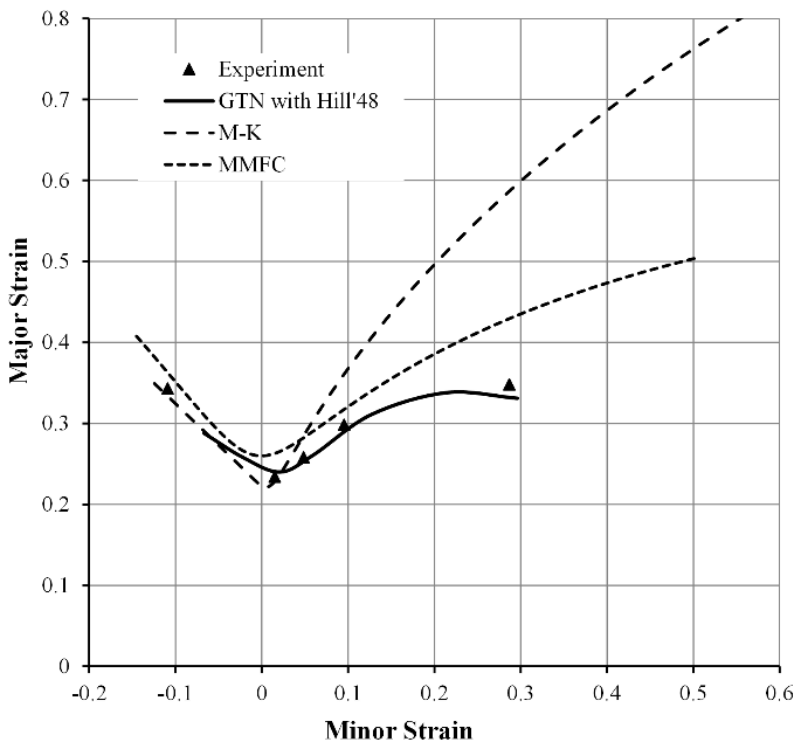

Fig. 3. Comparison of M-K, MMFC models with GTN models in terms of predicting experimental FLCs [17].

\subsection{FLC construction for sandwich sheets}


The FLC of a Bondal sandwich sheet was calculated using GTN model. The sandwich sheet has DC06 skins and a viscoelastic core layer, where the skins and the core layer are $0.6 \mathrm{~mm}$ and $0.05 \mathrm{~mm}$ thick, respectively. Mechanical properties of Bondal sheet layers and the GTN model parameters of DC06 skins are listed in Table 1.

Fig. 4 depicts the FLCs obtained by GTN and M-K models. This figure reveals that, GTN model predicts the strains in the middle and right side of the experimental FLC with good accuracy. But, it fails to predict the limit strains on the left side of the experimental FLC. On the other hand, as Fig. 4 suggests, the FLC calculated by the $\mathrm{M}-\mathrm{K}$ model coincides with the experimental forming limit strains in only two points. This poor prediction of M-K model might be originated from the fact that the value of thickness defect in M-K model is adjusted based on the limit strain associated with the plane strain point of the experimental FLC. However, from Fig. 4, one may conclude that GTN model provides more accurate FLCs compared to those of M-K model, as in the case of AA6016-T4 sheet (see also Fig. 3).

In order to study the effect of thickness and material of sandwich sheet layers on their formability, the FLCs of two sets of sandwiches were calculated using GTN model. Configurations of these sandwich sheets are presented in Table 2; where top skin refers to the skin sheet in contact with punch (in the Nakazima tests).

In Table 2, each sandwich sheet is designated as a four-character code in which the characters (from left to right) represent top skin material, bottom skin material, top skin thickness and bottom skin thickness, respectively. Furthermore, A, S, 1 and 2 stand for aluminum AA5754 alloy, mild-steel, thickness of $0.3 \mathrm{~mm}$ and thickness of $0.6 \mathrm{~mm}$, respectively. All sandwich sheets in Table 2 are equipped with a polymeric core layer of $0.6 \mathrm{~mm}$ thick. Mechanical properties and GTN parameters of the skins of the sandwiches are presented in Table 3.

Table 1. Mechanical properties of Bondal sheet layers and GTN model parameters of DC06 skins [25, 26].

\begin{tabular}{|c|c|c|c|c|c|c|}
\hline Material & Young Modulus, & Yield stress, & Tensile strength, & \multicolumn{3}{|c|}{ Lankford coefficient } \\
\cline { 4 - 7 } & {$[\mathrm{GPa}]$} & {$[\mathrm{MPa}]$} & $r_{0}$ & $r_{45}$ & $r_{90}$ \\
\hline DC06 & 210 & 152 & 279 & 2.027 & 1.751 & 2.467 \\
\hline Polymer & 8.8 & 15 & 61 & - & - & - \\
\hline- & \multicolumn{6}{|c|}{ GTN parameters } \\
\hline- & $f_{0}$ & $f_{N}$ & $S_{N}$ & $\bar{\varepsilon}_{N}^{p}$ & $f_{C}$ & $f_{F}$ \\
\hline DC06 & 0.0005 & 0.0008 & 0.1 & 0.3 & 0.0219 & 0.1677 \\
\hline
\end{tabular}

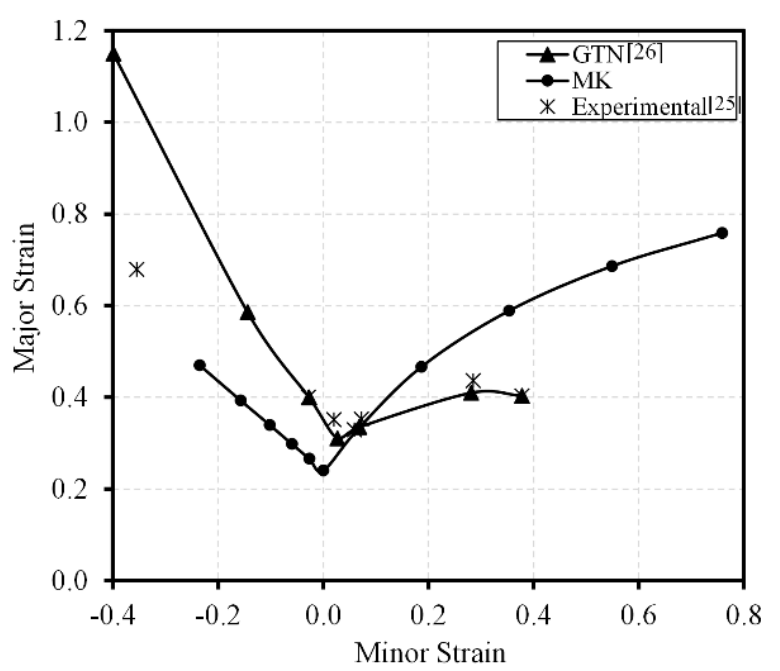

Table 2. Thickness and material of the metallic layers comprising the sandwich sheets.

\begin{tabular}{|c|c|c|c|}
\hline $\begin{array}{c}\text { Sandwich } \\
\text { sheet }\end{array}$ & $\begin{array}{c}\text { Sheet } \\
\text { name }\end{array}$ & $\begin{array}{c}\text { Material- } \\
\text { thickness (mm) } \\
\text { of top skin }\end{array}$ & $\begin{array}{c}\text { Material- } \\
\text { thickness (mm) } \\
\text { of bottom skin }\end{array}$ \\
\hline \multirow{4}{*}{ Set \#1 } & AA11 & A-0.3 & A-0.3 \\
\cline { 2 - 4 } & SS11 & S-0.3 & S-0.3 \\
\cline { 2 - 4 } & AS11 & A-0.3 & S-0.3 \\
\cline { 2 - 4 } & SA11 & S-0.3 & A-0.3 \\
\hline \multirow{5}{*}{ Set \#2 } & AS12 & A-0.3 & S-0.6 \\
\cline { 2 - 4 } & SA12 & S-0.3 & A-0.6 \\
\cline { 2 - 4 } & AS21 & A-0.6 & S-0.3 \\
\cline { 2 - 4 } & SA21 & S-0.6 & A-0.3 \\
\hline
\end{tabular}

Fig. 4. Comparison between M-K and GTN models in prediction of experimental FLC of the Bondal sandwich sheet $[25,26]$

Table 3. Mechanical properties and GTN parameters of the skins for the sandwiches categorized in set \#1 and set \#2 [27].

\begin{tabular}{|c|c|c|c|c|c|c|}
\hline \multirow[t]{2}{*}{ Material } & \multirow{2}{*}{$\begin{array}{c}\text { Young } \\
\text { Modulus, } \\
\text { [GPa] }\end{array}$} & \multirow{2}{*}{$\begin{array}{l}\text { Yield } \\
\text { stress, } \\
{[\mathrm{MPa}]}\end{array}$} & \multirow{2}{*}{$\begin{array}{c}\text { Ultimate } \\
\text { strength, } \\
\text { [MPa] }\end{array}$} & \multicolumn{3}{|c|}{ Lankford coefficient } \\
\hline & & & & $r_{0}$ & $r_{45}$ & $r_{90}$ \\
\hline Mild-steel & 198 & 148 & 389 & 2.20 & 1.90 & 1.60 \\
\hline AA5754 & 70 & 100 & 230 & 0.87 & 0.76 & 0.71 \\
\hline- & \multicolumn{6}{|c|}{ GTN parameters } \\
\hline- & $f_{0}$ & $f_{N}$ & $S_{N}$ & $\bar{\varepsilon}_{N}^{p}$ & $f_{C}$ & $f_{F}$ \\
\hline Mild-steel & 0.001 & 0.039 & 0.1 & 0.21 & 0.0601 & 0.1810 \\
\hline AA5754 & 0.001 & 0.034 & 0.1 & 0.32 & 0.0028 & 0.0977 \\
\hline
\end{tabular}


Total thickness of all sandwich sheets categorized in set \#1 was $1.2 \mathrm{~mm}$, with their skin thickness ratio being equal to one. These sandwich sheets are considered to evaluate the effect of layers material arrangement on the formability of the sandwiches. FLCs of the set \#1 sandwiches were calculated by numerical simulation of Nakazima tests using GTN model, as shown in Fig. 5. Clearly, SS11 had the highest forming limit, while the lowest forming limit belonged to SA11. The figure implies that, the alteration of layers material has a significant effect on the FLC. So that, by replacing the bottom skin of AA11 sheet with mild-steel (AS11), its formability will be improved. On the other hand, when the top skin of AA11 is substituted by mild-steel (SA11), a significant reduction will be observed in formability of the sandwich sheet. As in case of AA11 sheet, the minimum limit strain on the FLC is equal to 0.138 , which is $26.09 \%$ lower than that of AS11 (0.174) and 33.33\% higher than that of SA11 (0.092). The results illustrated in Fig. 4 are compatible with the reports of Sokolova et al. [28].

FLCs of the sandwich sheets in set \#2 were also calculated through GTN model. Categorized in set \#2 were the sandwich sheets composed of AS and SA with skin thickness ratio of 2 (or 0.5 ). FLCs of these sheets are also demonstrated in Fig. 6. This figure indicates that, formability of AS sheets is higher than that of SA sheets, which is in agreement with the results in Fig. 5. Moreover, Fig. 6 shows that AS12 and AS21 have almost the same formability (this holds true also for SA12 and SA21 sheets), implying that, in particular configurations of the sandwich sheet (e.g. AS), overall thickness of the sheet (rather than thickness ratio of the layers) is the dominant formability-controlling parameter. It should be noted that, this conclusion is just based on the results obtained at thickness ratio of 2 (or 0.5 ), and different results may be observed at higher thickness ratios [29].

\section{Conclusions}

Implementation of anisotropic GTN model with Hill'48 yield criterion and its application in FE simulation of square cup drawing and Nakazima tests were addressed in this work. Based on the results, the following conclusions can be drawn:

1- The results showed that GTN model is capable of correct fracture prediction in the square cup drawing tests. The drawing depth at the onset of fracture predicted by GTN model is $17.90 \mathrm{~mm}$, which was in a good agreement with the depth of $18.70 \mathrm{~mm}$ obtained in the experimental cup drawing.

2- GTN model allows for computation of limit strains on the FLC of AA6016-T4 aluminium sheet with good accuracy. The limit strains predicted by GTN model were more accurate than those of $\mathrm{M}-\mathrm{K}$ and MMFC models.

3- Once the bottom skin of AA-type sheets was replaced by mild-steel (AS-type sheet), its formability was improved. Contrarily, replacement of AA-type sheets top skin with mild-steel will result in formability decrease. When GTN model served as the basis, minimum limit strain of the FLC of AA11 sheet was found to be
0.138 , i.e. $26.09 \%$ lower than FLC of AS11 (0.174) and $33.33 \%$ higher than that of SA11 (0.092).

4- In case of AS12, AS21, SA12 and SA21 sheets, the arrangement of skin material was found as the dominant formability-controlling factor rather than the skin thicknesses. Moreover, for sandwich sheets of the same type (i.e. AS-type or SA-type), sheet formability was found to be controlled by overall thickness of the sandwich rather than thickness ratio of its layers.

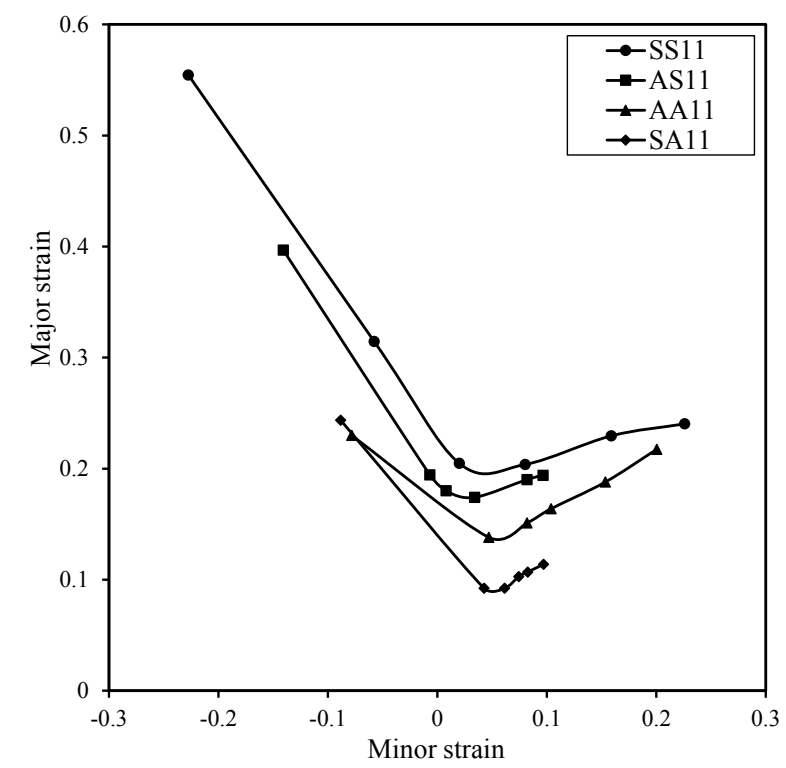

Fig. 5. The FLCs of the sandwich sheets of set \#1, calculated by GTN model [29].

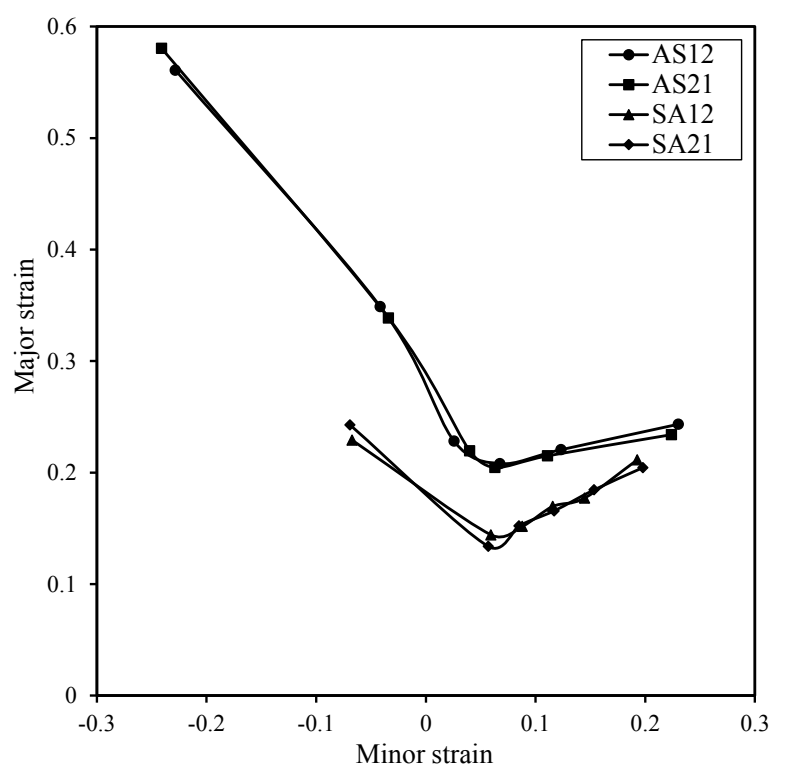

Fig. 6. FLCs of the sandwich sheets of set \#2, calculated by GTN model [29].

\section{References}

\footnotetext{
* Corresponding author: banabic@tcm.utcluj.ro
} 
1. F.A. McClintock, J. Appl. Mech. 35, 363-371 (1968).

2. J.R. Rice and D.M. Tracey, J. Mech. Phys. Solids 17, 201-217 (1969).

3. A.L. Gurson, J. Eng. Mater. Technol. 99, 2-15 (1977).

4. V. Tvergaard, Int J Fracture 17, 389-407 (1981).

5. V. Tvergaard, Int J Fracture 18, 237-252 (1982).

6. V. Tvergaard and A. Needleman, Acta Metall. 32, 157169 (1984).

7. D. Banabic, Multiscale Modelling in Sheet Metal Forming. Springer, 2016.

8. A.A. Benzerga and J. Besson, Eur. J. Mech. A. Solids 20, 397-434 (2001).

9. R. Hill, Proc R Soc Lond A-Math Phys Eng Sci 193, 281-297 (1948).

10. V. Monchiet, C. Gruescu, E. Charkaluk, and D. Kondo, Comptes Rendus Mécanique 334, 431439 (2006).

11. V. Monchiet, O. Cazacu, E. Charkaluk, and D. Kondo, Int. J. Plast. 24, 1158-1189 (2008).

12. S.M. Keralavarma and A.A. Benzerga, Comptes Rendus Mécanique 336, 685-692 (2008).

13. S.M. Keralavarma and A.A. Benzerga, J. Mech. Phys. Solids 58, 874-901 (2010).

14. L. Morin, J.-B. Leblond, and D. Kondo, Int J Solids Struct. 77, 86-101 (2015).

15. J.B. Stewart and O. Cazacu, Int J Solids Struct. 48, 357-373 (2011).

16. O. Cazacu, B. Plunkett, and F. Barlat, Int. J. Plast. 22, 1171-1194 (2006).
17. A. Kami, B.M. Dariani, A. Sadough Vanini, D.S. Comsa, and D. Banabic, J. Mater. Process. Technol. 216, 472-483 (2015).

18. K.C. Liao, J. Pan, and S.C. Tang, Mech. Mater. 26, 213-226 (1997).

19. D.A. Wang, J. Pan, and S.D. Liu, Int J Damage Mech 13, 7-33 (2004).

20. Z. Chen and X. Dong, Comp Mater Sci 44, 1013-1021 (2009).

21. F. Barlat and K. Lian, Int. J. Plast. 5, 51-66 (1989).

22. A. Kami, B. Mollaei Dariani, A. Sadough Vanini, D.S. Comsa, and D. Banabic, Proc Rom Acad Ser A 15, 300-309 (2014).

23. A. Bragard, Baret, J.C., Bonnarens, H., Rapp. Cent. Rech. Metall. 33, (1972).

24. R. D'Haeyer, Bragard, A., Rap. Cent. Rech. Metall. 42, (1975).

25. A. Kami, B.M. Dariani, D.S. Comsa, D. Banabic, A.S. Vanini, and M. Liewald, Proc Rom Acad Ser A 18, 281-290 (2017).

26. A. Kami, B. Mollaei Dariani, D.S. Comsa, D. Banabic, A. Sadough Vanini, and M. Liewald, Rom. J. Techn. Sci. Appl. Mechanics 61, 248264 (2016).

27. M. Brunet, S. Mguil, and F. Morestin, J. Mater. Process. Technol. 80-81, 40-46 (1998).

28. O. Sokolova, A. Carradó, and H. Palkowski, Adv. Mater. Res. 137, 81-128 (2010).

29. A. Kami, K. Chung, and D. Banabic, Rom. J. Techn. Sci. Appl. Mechanics 62, 30-39 (2017). 